\title{
Escherichia coli from Nigeria exhibit a high prevalence of antibiotic resistance where reliance on antibiotics in poultry production is a potential contributing factor
}

\author{
Chijioke A. Nsofor ${ }^{1,}$, Isaac O. Olatoye ${ }^{2,4}$, Elizabeth A. Amosun ${ }^{3}$, Christian U. Iroegbu ${ }^{1}$, \\ Margaret A. Davis ${ }^{4}$, Lisa H. Orfe ${ }^{5}$ and Douglas R. Call ${ }^{4,5 *}$ \\ ${ }^{1}$ Department of Microbiology, University of Nigeria, Nsukka, Nigeria. \\ ${ }^{2}$ Department of Veterinary Public Health and Preventive Medicine, University of Ibadan, Ibadan, Nigeria. \\ ${ }^{3}$ Department of Veterinary Microbiology and Parasitology, University of Ibadan, Nigeria. \\ ${ }^{4}$ Paul G. Allen School for Global Animal Health, Washington State University, Pullman, WA, USA. \\ ${ }^{5}$ Department of Veterinary Microbiology and Pathology, Washington State University, Pullman, WA, USA.
}

Accepted 12 September, 2013

\begin{abstract}
To assess the prevalence of antibiotic resistance in Nigeria, single Escherichia coli isolates were collected from a geographically diverse panel of fecal samples collected from human clinical and nonclinical donors $(n=77)$, livestock (cattle, swine, and goats) and chickens $(n=71$ total). There was no difference in the proportion of isolates resistant to $\geq 1$ antibiotics from human clinical and non-clinical samples, but overall, this was significantly higher for human $(85.7 \%)$ compared to animal $(53.5 \%)$ isolates $(P<0.0001)$. The average number of resistance phenotypes per isolate was significantly higher for human (5.0), goat (4.0), and poultry (3.4) compared with cattle (2.4) and swine (2.0) $(P<0.05)$. There were 25 different resistance phenotypes with more diversity from animal compared with human isolates. A survey of management practices at $\mathbf{3 0}$ poultry farms in the vicinity of lbadan found that all respondents self-milled feed and most $(87.7 \%)$ routinely added antibiotics to feed. Tetracyclines were the dominant antibiotics of choice followed by tylosin and gentamicin and some use of chloramphenicol, ciprofloxacin, and enrofloxacin. If this pattern of antibiotic resistance and use is repeated across the different sectors of food-animal production and in multiple developing countries, then trade and travel are likely to disseminate resistance traits to other countries potentially negating local policies that are designed to limit selection for antibiotic resistant bacteria.
\end{abstract}

Key words: Antibiotic resistance, Escherichia coli, growth promotion, poultry, chloramphenicol.

\section{INTRODUCTION}

Antibiotic resistance is a significant worldwide public health concern (French, 2010; Kumarasamy et al., 2010) both because of resistance found in specific pathogens (Delsol et al., 2004; Hurd et al., 2010), and because resistance harbored by commensal organisms may serve as a reservoir of traits that can be disseminated to pathogens (Pallecchi et al., 2007; Shoemaker et al., 2001;
$\mathrm{Li}$ and Wang, 2010). Antibiotic resistant bacteria that normally pose no immediate disease risk can also become opportunistic pathogens that complicate postoperative recovery (Patel and Kirby, 2008) or otherwise become problematic for immunocompromised individuals (Bonomo, 2001). While a number of ecological factors probably contribute to the dissemination and maintenance 
of antibiotic resistance traits, it is clear that antibiotic use is a primary factor that selects for the evolution and amplification of antibiotic resistant bacteria in humans and animals.

The rising prevalence of antibiotic resistance is a particularly important problem in deve-loping countries where there is limited control of the quality, distribution and use of antibiotics in human medicine, veterinary medicine, and food-animal agricul-ture (Okeke et al., 1999). The relative contribution of these three sectors to the evolution and amplification of antibiotic resistance is not clearly understood (McDermott et al., 2002; Oliver et al., 2011) although it probably varies with different countries. Indeed, many of the highly complex issues in developing countries that influence antibiotic use practices in human medicine (Okeke et al., 1999) are likely to apply to both veterinary and food-animal applications.

At a broader level, the evolution and amplification of antibiotic resistance traits in developing countries should be a significant focus of attention for industrialized countries as well. For example, while policy makers in the USA debate the merits of limiting antibiotic use in food animals (DHHS, 2010b), the potential benefits of such limits could be overshadowed by amplification in developing countries and dissemination of antibiotic resistant bacteria and resistance traits through travel and trade. More efforts are needed to understand how antibiotic use practices at an international scale ultimately influence the prevalence and dissemination of antibiotic traits within individual countries.

Bacterial antibiotic resistance has been highlighted in Nigeria through a series of published studies since the 1990's (Okeke et al., 1999; Fashae et al., 2010; Akinyemi et al., 2011; Ogbolu et al., 2011) and thus Nigeria provides a model for considering how antibiotic use in different sectors of a developing country can impact the prevalence of antibiotic resistant bacteria. Our first objective was to conduct a comparative analysis of antibiotic resistance for bacteria from humans and domestic animals under the null hypothesis that the prevalence of antibiotic resistant bacteria would be equal for human and animal populations in Nigeria. We focused on $E$. coli because this species includes a broad range of both pathogenic and commensal serovars that can harbor antibiotic resistance traits of interest to human and veterinary medicine. Our second objective was to survey antibiotic use practices in poultry production. Widespread consumption of poultry by Nigerian consumers makes this sector of food production a potentially important source of antibiotic resistant bacteria in the human diet.

\section{MATERIALS AND METHODS}

\section{Sample collection}

The study population included humans (who were either ill or presumptively healthy) and a variety of presumptively healthy domestic livestock viz. cattle, goats, pigs and chicken obtained from five geopolitical zones of Nigeria viz. south-east, south-west, southsouth, north-central and north-north. In the south-south and southeast, clinical specimens were collected at the University of Port Harcourt Teaching Hospital, Port Harcourt, Rivers State, and the Abia State University Teaching Hospital, Aba, Abia State, respectively. The Lagos State University Teaching Hospital, Ikeja, Lagos, was the site of specimen collection for the south-west, while the National Hospital, Abuja and Military Reference Hospital, Kaduna State, were the sources of specimens from the northcentral and north-north, respectively. All fecal samples from these hospitals were clinical specimens from patients presenting with gastroenteritis. Fecal samples were also collected from apparently healthy undergraduate students at Madonna University Elele, Rivers State. For this convenience sample, individuals were limited to those reporting no exposure to antibiotics for six months prior to sampling and each person received an explanation of the study objectives and consent form for inclusion in the study.

All sampling procedures were in accordance with guidelines that are promulgated by the National Health Research Ethics Committee in Nigeria (www.nhrec.net). None of the animals included in this study (at the time of specimen collection) exhibited signs and symptoms of abnormal health. The cattle and goat specimens came from the Obinze livestock market Owerri, Imo State, while the Madonna University Poultry Elele, Rivers State, was the source of poultry specimens. The specimens from swine came from a farm located at the Ogbor-Hill area of Aba, Abia State. There was no documented evidence of antibiotics use in the farms from which the specimens were collected, although the manager of the poultry farm indicated occasional antibiotic use at this facility. In all, a single non-duplicate $E$. coli isolate was selected from each human and animal fecal sample to maximize biological independence between observations.

\section{Isolation of $E$. coli}

Fresh fecal droppings were collected from animals and care was taken to avoid collecting more than one fecal sample per individual animal. Feces were packed in a sterile plastic container and were transported to the laboratory on ice for processing and cultivation. 1 $\mathrm{g}$ of each animal's feces was diluted in $9 \mathrm{ml}$ of $0.85 \%$ sterile saline solution. The contents were mixed thoroughly and serially diluted (10-fold) before plating on Eosin Methylene Blue agar (EMB) (Oxoid, England) to isolate Gram-negative enteric bacilli (HoltHarris and Teague, 1916; Levine, 1918). Human fecal specimens were streaked directly on EMB agar with a sterile inoculating loop. No antibiotic was included in the EMB agar plates used for cultivation. The inoculated plates were incubated overnight at $37^{\circ} \mathrm{C}$. A single isolate resembling $E$. coli (green metallic sheen on EMB) was selected from an individual fecal sample for further characterization. Identification of $E$. coli was confirmed using conventional microbiological tests; indole positive, methyl-red positive and citrate negative (Cheesbrough, 2000). Isolates were transported to Washington State University Field Diseases Investigation Unit (WSU-FDIU, Pullman, WA) where identity was further confirmed by characteristic growth on 4-methylumbelliferylb-D-glucuronide (MUG) supplemented with violet red bile (VRBMUG) (Venkateswaran et al., 1996) and by an indole test.

\section{Antibiotic susceptibility testing}

The antibiotic susceptibility pattern of the isolates was determined using the disk diffusion method on Mueller-Hinton agar at the WSUFDIU. Inhibition zone sizes were interpreted using standard recommendations of the Clinical Laboratory Standard Institute (CLSI, 2008). Susceptibility was tested against ampicillin (AM; $10 \mathrm{ug}$ ), 
Table 1. Percentage of antibiotic resistant E. coli from humans and animals in Nigeria.

\begin{tabular}{lcccccccc}
\hline \multirow{3}{*}{ Antibiotic } & \multicolumn{3}{c}{ Human } & \multicolumn{5}{c}{ Animal } \\
\cline { 2 - 9 } & $\begin{array}{c}\text { Clinical } \\
(\mathbf{n = 6 5 )}\end{array}$ & $\begin{array}{c}\text { Non-clinical } \\
(\mathbf{n}=\mathbf{1 2})\end{array}$ & $\begin{array}{c}\text { Human combined } \\
(\mathbf{n}=\mathbf{7 7})\end{array}$ & $\begin{array}{c}\text { Cattle } \\
(\mathbf{n}=\mathbf{2 7})\end{array}$ & $\begin{array}{c}\text { Goats } \\
(\mathbf{n}=\mathbf{1 3})\end{array}$ & $\begin{array}{c}\text { Poultry } \\
(\mathbf{n}=\mathbf{1 4})\end{array}$ & $\begin{array}{c}\text { Swine } \\
(\mathbf{n}=\mathbf{1 7})\end{array}$ & $\begin{array}{c}\text { Animal combined } \\
(\mathbf{n}=\mathbf{7 1})\end{array}$ \\
\hline $\mathrm{Am}^{\mathrm{a}}$ & 78.5 & 83.3 & 79.2 & 29.6 & 61.5 & 64.3 & 35.3 & 43.7 \\
$\mathrm{Amc}$ & 32.3 & 41.7 & 33.8 & 14.8 & 30.8 & 28.6 & 5.9 & 18.3 \\
$\mathrm{An}$ & 0.0 & 0.0 & 0.0 & 3.7 & 0.0 & 0.0 & 0.0 & 1.4 \\
$\mathrm{C}$ & 23.1 & 8.3 & 20.8 & 18.5 & 23.1 & 21.4 & 5.9 & 16.9 \\
$\mathrm{Caz}$ & 1.5 & 0.0 & 1.3 & 0.0 & 0.0 & 7.1 & 0.0 & 1.4 \\
$\mathrm{G}$ & 83.1 & 91.7 & 84.4 & 37.0 & 69.2 & 64.3 & 35.3 & 47.9 \\
$\mathrm{Gm}$ & 13.8 & 16.7 & 14.3 & 11.1 & 0.0 & 0.0 & 5.9 & 5.6 \\
$\mathrm{~K}$ & 12.3 & 16.7 & 13.0 & 11.1 & 0.0 & 0.0 & 5.9 & 5.6 \\
$\mathrm{Nal}$ & 24.6 & 25.0 & 24.7 & 18.5 & 0.0 & 14.3 & 5.9 & 11.3 \\
$\mathrm{~S}$ & 67.7 & 66.7 & 67.5 & 22.2 & 61.5 & 35.7 & 29.4 & 33.8 \\
$\mathrm{Sxt}$ & 83.1 & 83.3 & 83.1 & 37.0 & 69.2 & 57.1 & 35.3 & 46.5 \\
$\mathrm{~T}$ & 81.5 & 83.3 & 81.8 & 37.0 & 84.6 & 50.0 & 35.3 & 47.9 \\
\hline
\end{tabular}

${ }^{\mathrm{a}} \mathrm{Am}=$ ampicillin; Amc = amoxicillin/clavulanic acid; $\mathrm{An}=$ amikacin; $\mathrm{C}=$ chloramphenicol; $\mathrm{Caz}=$ ceftazidime; $\mathrm{G}=$ sulfisoxazole; $\mathrm{Gm}=$ Gentamicin; K = kanamycin; Nal = nalidixic acid; S = streptomycin; Sxt = trimethoprim/sulfamethoxazole; and T = tetracycline.

amikacin (AN; $10 \mathrm{ug}$ ), amoxicillin/clavulanic acid (AmC; 20/10 ug), chloramphenicol (C; $30 \mathrm{ug})$, ceftazidime (CAZ; $30 \mathrm{ug})$, sulfisoxazole (G; $0.25 \mathrm{mg}$ ), gentamycin (GM; $10 \mathrm{ug})$, kanamycin (K; $30 \mathrm{ug})$, nalidixic acid (NA; $30 \mathrm{ug})$, streptomycin (S; $10 \mathrm{ug})$, sulfamethaxazole/trimethoprim (SxT; $23.75 \mathrm{ug} / 1.25 \mathrm{ug})$, and tetracycline (TE; $30 \mathrm{ug}$ ). All antibiotic discs were purchased from Becton Dickinson (Franklin Lakes, NJ). E. coli (ATCC 25922), Staphylococcus aureus (ATCC 25932), and Enterococcus fecalis (ATCC 29212) were used as reference strains for culture identification and antibiotic susceptibility testing.

\section{Pulsed-field gel electrophoresis (PFGE)}

PFGE was performed using Xbal (New England Biolabs) based on the PulseNet protocol with minor modifications (www.cdc.gov/pulsenet/protocols.htm). Briefly, DNA fragments were resolved by electrophoresis in 1\% SeaKem Gold agarose gels with a CHEF DRII machine (Bio-Rad), using 0.5X Tris-borate-EDTA as the buffer. Gels were run for $18 \mathrm{~h}$ at $14^{\circ} \mathrm{C}$, using a linearly ramped switching time from $2.2 \mathrm{~s}$ to $63.8 \mathrm{~s}$ and a voltage of $6.0 \mathrm{~V} / \mathrm{cm}^{2}$. After electrophoresis, the gels were stained in $400 \mathrm{ml}$ of deionized water containing $40 \mathrm{ul}$ of $10 \mathrm{mg} / \mathrm{ml}$ of ethidium bromide for $20 \mathrm{~min}$ on a rocker and destained three times for $20 \mathrm{~min}$ each with distilled water. Bands were visualized by a UV transilluminator (Fisher Scientific) and photographed using an Alpha imager (Alpha Innotech Corporation, San Leandro, CA, USA). Digitalized gel images were analyzed using BioNumerics software version 4.0 (Applied Maths, Sint-Martens-Latem, Belgium). Cluster analysis was performed by using the Unweighted Pair Group Method using arithmetic Averages (UPGMA) based on Dice coefficients to quantify the similarities.

\section{Survey of poultry producers}

During an eight week survey between April and June 2009, semistructured questionnaires were administered to 30 poultry farmers randomly selected from six local governing areas of the Ibadan metropolis. Data was obtained for management practices including commonly used antibiotics, indications for use, sources and knowledge of withdrawal period and antibiotic resistant food-borne pathogens. Invariably, it was not possible to provide precise case definitions or diagnostic confirmation, but self-reported diseases could be characterized into ten categories.

\section{RESULTS}

\section{Antibiotic resistance}

In total, we collected a single isolate of $E$. coli from each of 77 human and 71 animal fecal samples (Table 1). There was less overall variance in the proportion of resistant isolates for human clinical and non-clinical isolates (average $4.1 \%$ difference) compared with the proportion of resistant isolates between different animal hosts (average 23.7\% difference) (Table 1). The prevalence of resistance ranged from nearly zero for amikacin to a high of $84.4 \%$ for human isolates resistant to sulfisoxazole and a high of $47.9 \%$ for animal isolates resistant to sulfisoxazole or tetracycline. We detected resistance to ceftazidime (a third-generation cephalosporin) in one poultry isolate (7.1\%).

Sample sizes precluded analysis across individual antibiotics, but it is notable that there was no statistical difference in the proportion of isolates that were resistant to $\geq 1$ antibiotic for human clinical (84.6\%) and nonclinical $(91.7 \%)$ isolates (Fisher's exact test, $P=1.0$ ). Human isolates were more likely to be resistant to $\geq 1$ antibiotic compared with animal isolates (85.7 and $53.5 \%$, respectively, Fisher's exact test, $P<0.0001)$. The average number of resistance phenotypes per isolate was significantly higher for human (5.0), goat (4.0) and poultry (3.4) compared with cattle (2.4) and swine (2.0) (one-way ANOVA, $P<0.0001$; Tukey-Kramer multiple comparison test, $P<0.05)$. 
Table 2. Distribution of multidrug resistance phenotypes amongst human and animal isolates collected in Nigeria.

\begin{tabular}{|c|c|c|c|c|}
\hline \multirow{2}{*}{ Antibiotic resistance phenotype } & \multicolumn{2}{|c|}{ Human } & \multicolumn{2}{|c|}{ Animal } \\
\hline & Number & Percent (\%) & Number & Percent (\%) \\
\hline None & 11 & 14.3 & 33 & 46.5 \\
\hline $\mathrm{Am}^{\mathrm{a}}$ & 0 & 0.0 & 1 & 1.4 \\
\hline AmCGmKSxtSTAmcNalG & 1 & 1.3 & 1 & 1.4 \\
\hline AmCGmKSxtSTNalG & 1 & 1.3 & 0 & 0.0 \\
\hline AmCGmSxtSTNalG & 2 & 2.6 & 0 & 0.0 \\
\hline AmCSxtSG & 0 & 0.0 & 2 & 2.8 \\
\hline AmCSxtSTAmcG & 0 & 0.0 & 3 & 4.2 \\
\hline AmCSxtSTAmcNalG & 2 & 2.6 & 1 & 1.4 \\
\hline AmCSxtSTG & 3 & 3.9 & 0 & 0.0 \\
\hline AmCSxtSTNalG & 4 & 5.2 & 2 & 2.8 \\
\hline AmGmKAnSxtSTAmcNalG & 0 & 0.0 & 1 & 1.4 \\
\hline AmGmKSxtSTAmcNalG & 6 & 7.8 & 1 & 1.4 \\
\hline AmGmKSxtSTNalG & 1 & 1.3 & 0 & 0.0 \\
\hline AmGmKSxtTAmcNalG & 0 & 0.0 & 1 & 1.4 \\
\hline AmKSxtSAmcNalGCaz & 1 & 1.3 & 0 & 0.0 \\
\hline AmSxtSNalG & 1 & 1.3 & 0 & 0.0 \\
\hline AmSxtSTAmcG & 15 & 19.5 & 1 & 1.4 \\
\hline AmSxtSTG & 15 & 19.5 & 11 & 15.5 \\
\hline AmSxtTAmcG & 1 & 1.3 & 3 & 4.2 \\
\hline AmSxtTAmcNalGCaz & 0 & 0.0 & 1 & 1.4 \\
\hline AmSxtTG & 8 & 10.4 & 1 & 1.4 \\
\hline AmTG & 0 & 0.0 & 1 & 1.4 \\
\hline CSxtTG & 3 & 3.9 & 3 & 4.2 \\
\hline $\mathrm{G}$ & 1 & 1.3 & 0 & 0.0 \\
\hline SxtSG & 0 & 0.0 & 1 & 1.4 \\
\hline $\mathrm{T}$ & 1 & 1.3 & 3 & 4.2 \\
\hline Total & 77 & 100 & 71 & 100 \\
\hline
\end{tabular}

${ }^{\mathrm{a}} \mathrm{Am}=$ ampicillin; $\mathrm{Amc}=$ amoxicillin/clavulanic acid; $\mathrm{An}=$ amikacin; $\mathrm{C}=$ chloramphenicol; $\mathrm{Caz}=$ ceftazidime; $\mathrm{G}=$ sulfisoxazole; $\mathrm{Gm}=$ Gentamicin; K = kanamycin; Nal = nalidixic acid; S = streptomycin; Sxt = trimethoprim/sulfamethoxazole; and T = tetracycline.

We detected 26 different resistance phenotypes with 22 having $>1$ trait (Table 2). Approximately $50 \%$ of human isolates were classified into one of three resistance phenotypes that included a core resistance to ampicillin, trimethoprim/sulfamethoxazole, tetracycline and sulfixoxazole. Resistance phenotypes for animals were more diverse with the largest proportion (15.5\%) sharing the same core resistance traits as described above with the addition of resistance to streptomycin (Table 2). The PFGE analysis showed that animal isolates were interspersed without any clear host association (data not shown). Interestingly, two clusters of human isolates ( $n=18$ and $n=15$ isolates) were mostly distinct from animal isolates and included both human clinical and non-clinical E. coli. Both clusters were comprised of isolates from geographically separated parts of the country indicating that there was no spatial separation in the distribution of antibiotic resistant $E$. coli from humans. Using an arbitrarily selected $85 \%$ similarity threshold for the PFGE data grouped $52.4 \%$ of isolates into clusters having $\geq 3$ isolates. A slight majority of human isolates $(53.3 \%)$ were found in human dominated clusters $(>50 \%)$ while only $38.0 \%$ of animals isolates were found in animal dominated clusters.

\section{Poultry practices survey}

In total we interviewed 30 poultry producers to assess their animal husbandry and antibiotic use practices. The majority of producers had primary $(16.7 \%)$ or secondary (36.7\%) education with an additional $20 \%$ having earned a secondary education diploma or certificate. The remaining farmers had some university experience $(13.3 \%)$ while $2(6.7 \%)$ were veterinarians. Two other producers did not disclose their education experience. The majority of producers $(64 \%)$ had $>10$ years of poultry farming experience, with an average of 14.6 years. 


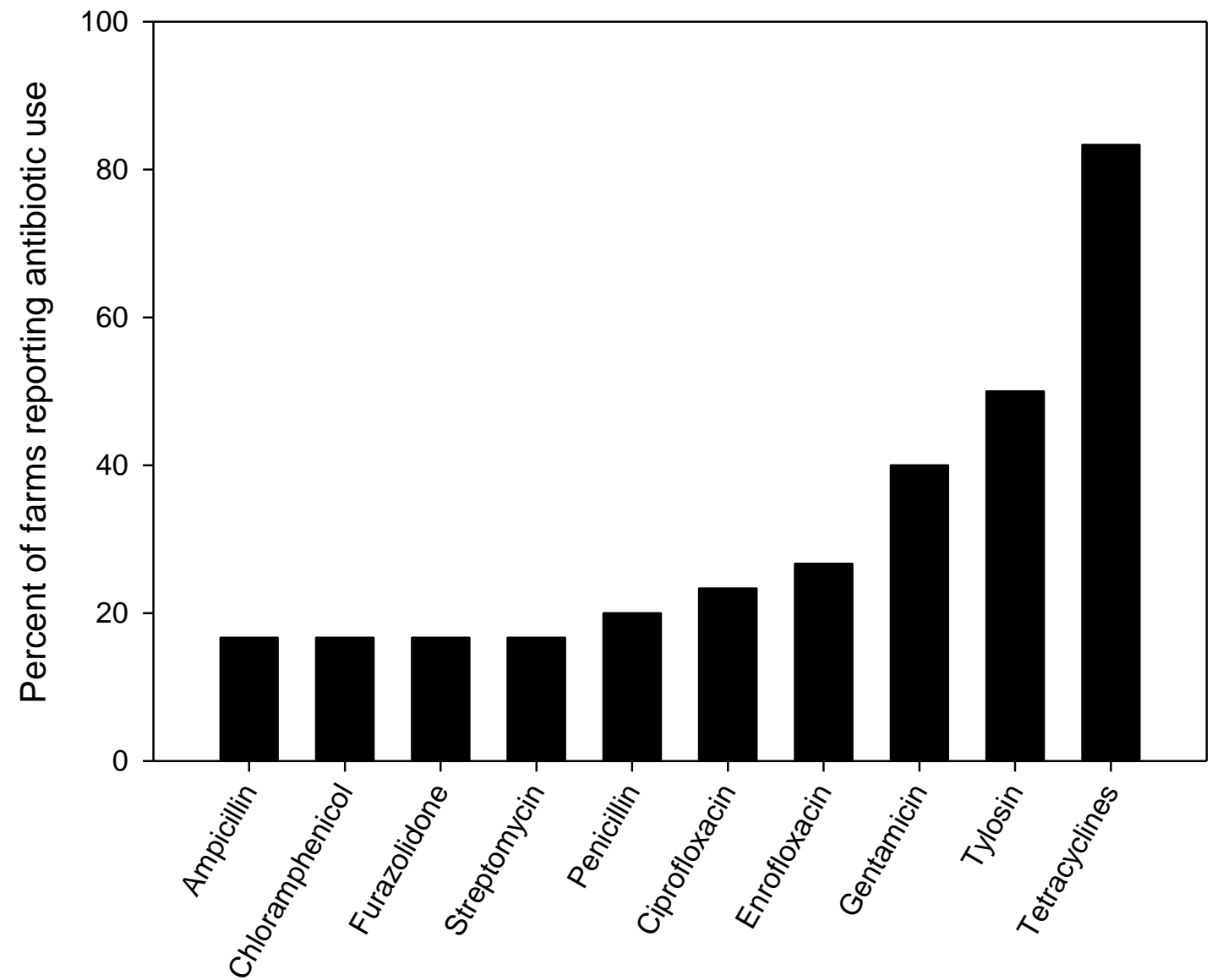

Figure 1. Percent of Nigerian poultry producers ( $n=30$ total) reporting use of different antibiotics.

Sixty percent $(n=18)$ of farmers practiced a "deep litter" system while $23.3 \%(n=7)$ practiced both deep litter and a "battery cage" system to house their flocks. The median flock population was 4,500 (range $=250$ to 65,000 ) birds with $83.3 \%$ of farmers considered "largescale" producers. All the respondents engaged in selfmilling of feed for their flocks and all farms reported use of at least two antibiotics. The majority of producers used at least three antibiotics $(n=15)$ while seven other producers reported using between four and six different antibiotics. The majority of producers $(87.7 \%)$ routinely added antibiotics to the feed for disease prevention and to improve production. Tetracyclines (oxytetracycline and chlortetracycline) were the most commonly used antibiotic (Figure 1). Approximately, $50 \%$ of producers also used gentamicin while approximately $20 \%$ of producers employed fluoroquinolones (ciprofloxacin or enrofloxacin) and chloramphenicol. A majority of respondents $(86.7 \%)$ did not engage the services of a veterinarian for disease diagnosis or for drug prescriptions and use. Records of diseases and treatments were not available in most of the surveyed farms. A majority of producers (63.3\%) were not aware of or did not adhere to antibiotic withdrawal periods before selling poultry products for human consumption. Producers typically purchased antibiotics from retail sources.

To better understand the motivation for antibiotic use, we also attempted to identify the most common infectious disease challenges that are faced by poultry producers and the history of antibiotic use in the flocks. The majority $(93.3 \%)$ of the farmers diagnosed disease conditions based on clinical signs and post mortem findings while $6.7 \%$ engaged laboratory confirmation and antibiotic sensitivity tests for bacterial isolates. There was a diverse array of infectious disease challenges including chronic respiratory disease (etiology unknown) and more specific bacterial and viral diseases (Figure 2).

\section{DISCUSSION}

One of the strengths of the current study is that we restricted our analysis to only one $E$. coli isolate per fecal sample thereby maximizing biological independence between isolates and thereby limiting bias from any single individual. The independence between isolates was consistent with the generally high diversity of PFGE 


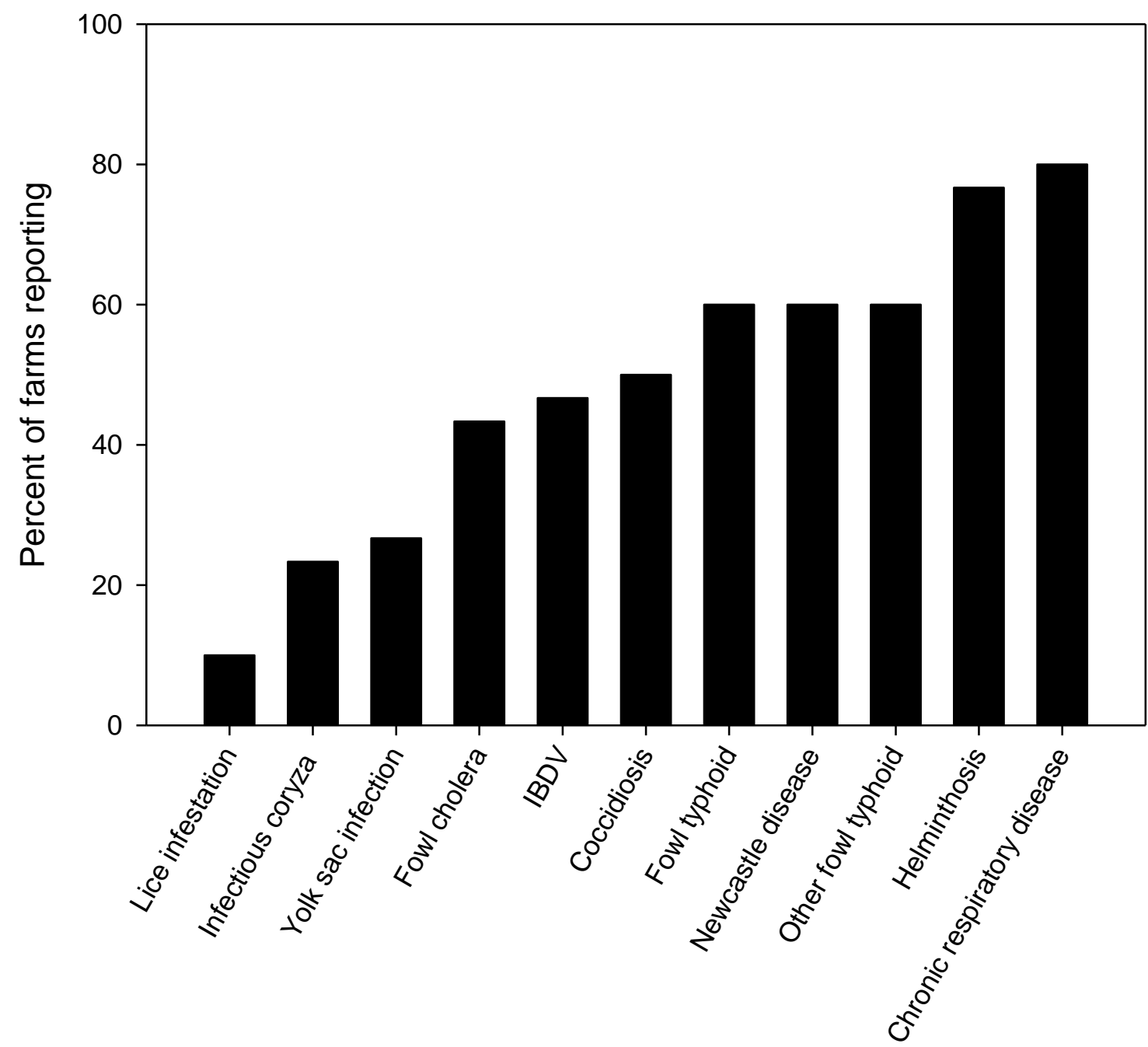

Figure 2. Percent of Nigerian poultry producers ( $n=30$ total) reporting occurrence of different infectious disease or parasite problems. Disease classifications include chronic respiratory disease (cough; suspect Mycoplasma); helminthosis (parasitic worm infestation); Newcastle disease (suspect avian paramyxovirus); coccidiosis (bloody diarrhea caused by bacterium or protozoa); infectious bursal disease virus (IBDV); fowl cholera (pasteurellosis); yolk sac infection (salmonellosis or colibacillosis); infectious coryza (suspect Hemophilus paragallinarum); other fowl typhoid (suspect non-bloody diarrhea salmonellosis); lice infestation.

profiles detected in this study. Human isolates were mostly distinct from animal isolates by PFGE, and the human isolates were collected from multiple geographic locations suggesting that there are probably distinct sources of $E$. coli that colonize humans while animal isolates are more diverse in origin. It is also important to note that human clinical isolates can represent a potential source of bias because isolates collected at hospitals may have been enriched in numbers due to antibiotic treatment prior to collection. If this was a significant bias in our study we would expect the proportion of resistant strains to be significantly lower for non-clinical isolates. Nevertheless, while our non-clinical isolate collection was limited in number, the proportion of antibiotic resistant $E$. coli was not different from clinical samples.
Overall, it is clear that $E$. coli isolates from humans were disproportionately more resistant to antibiotics $(85.7 \%)$ compared with $E$. coli isolates from animals $(53.5 \%)$. Furthermore, the majority of antibiotic resistant isolates expressed multidrug resistance phenotypes (97 and $89.5 \%$ for human and animal isolates, respectively). While it is impossible, from our data, to estimate selection coefficients for different antibiotic resistance traits, the difference in proportion of resistance from human and animal isolates is consistent with a higher degree of selection in the human population. It is also notable that the $E$. coli collected from livestock and poultry came from adult- or market-age animals and is generally higher than reported for similar studies in developed countries (Khachatryan et al., 2004; Sato et al., 2005; Cho et al., 2007). 
Thus, the high level of resistance documented for livestock and poultry in the current study is not biased by the tendency for younger animals to harbor disproportionately more resistant $E$. coli. On average, resistance among animal isolates was ordered with swine $<$ cattle $<$ poultry < goat. This finding combined with the diversity of resistance phenotypes across different animal groups (Table 1) is consistent with distinct ecologies and husbandry practices between different food animal sectors, although unequal sampling of animal populations across geographic space is a confounding factor in this study. Regardless, our samples were collected from facilities that purportedly used little or no antibiotics. If correct, our findings highlight the fact that once antibiotic resistant traits are circulating in a population, a variety of factors may favor their long-term persistence even in the absence of antibiotic selection pressure (Khachatryan et al., 2006; Singer et al., 2006).

The Nigerian livestock industry provides about $94 \%$ of the animal protein that is consumed in Nigeria and this sector of the economy contributes $4-5 \%$ of the national gross domestic product (FGN, 2009). The poultry sector accounts for about $25 \%$ of local meat production in Nigeria and poultry represents a source of quality protein and employment for a sizeable proportion of the populace. About $20 \%$ of the poultry population in Nigeria is reared within intensive commercial systems, most of which are located in the southwestern Nigeria with lbadan being the main entry point for imported poultry products. This is also the location of the major poultry breeders and retailers that distribute poultry around the country and beyond (Owoade et al., 2004). Consequently, poultry represents an important component of the Nigerian diet and a potentially important source of selection for antibiotic resistant bacteria.

Our surveys found that the majority of the poultry farmers routinely used antibiotics as feed additives to prevent and treat infections and to boost production without veterinarian oversight. These practices, which appear to be common among developing countries (Mitema et al., 2001; Kabir et al., 2004), contribute to both selection for antibiotic resistance and probably introduce antibiotic residues into local food products (Riviere and Spoo, 1995). The most widely used antibiotic reported by poultry farmers was a tetracycline compound, which is consistent with other studies in developing countries (Al-Ghamdi et al., 2000; Nonga et al., 2010). Respondents to our survey indicated that they used several antibiotics that could be problematic from a public health perspective. For instance, the U.S. Food and Drug Administration has prohibited use of chloramphenicol in food animals (DHHS, 2010a) in part because residues in meat products can cause aplastic anemia and other blood disorders in humans (Fraunfelder et al., 1993; Young and Alter, 1994). Consequently, this practice represents a genuine public health risk beyond selecting for antibiotic resistant bacteria and it will certainly contribute to trade complications. A number of producers also used either ciprofloxacin or enrofloxacin, both of which are fluoroquinolones. In the U.S, fluoroquinolones have been banned from use in poultry primarily because of the potential to select for ciprofloxacin resistant Campylobacter jejuni (Delsol et al., 2004; DHHS, 2005; Hurd et al., 2010). In general, fluoroquinolone use should be limited whenever possible because of the high probability of de novo resistance arising from simple chromosomal point mutations. There is also growing evidence of proliferation of quinolone resistance in Nigeria including detection of horizontally transmitted resistance traits (Aypak et al., 2009; Fortini et al., 2011).

It is important to note that the Nigerian poultry industry is constantly faced with challenges of high input costs, low egg production, diseases and pests, low and poor performing breeds, poor weight gain/feed conversion, feeding and management problems and lack of capital (Isiaka, 1998; van Eekeren et al., 2004). Fowl typhoid is one example of a significant problem for poultry producers in developing countries; in Nigeria an estimated $18.4 \%$ of flocks are affected (Mbuko et al., 2009). Prevention and treatment of salmonellosis and other bacterial infections are major challenges of profitable poultry production and this is a primary motivation for the heavy reliance on antibiotics (Kabir et al., 2004). In developing countries including Nigeria, antibiotics are easily acquired and used without veterinary oversight (Dina and Arowolo, 1991; Kabir et al., 2004). While potentially important to sustain production, these practices most likely contribute to development and expansion of antibiotic resistance.

In 2001, the WHO launched the first global strategy to develop antibiotic use and resistance surveillance programs and networks across several regions of the world (Simonsen et al., 2004). There is no coordinated effort, however, to monitor or control antibiotic resistance in Nigeria and there is no coordinated intervention effort to provide resources and education to livestock and poultry producers to help them limit their need for antibiotics. Clearly, there are multiple opportunities for meaningful interventions to improve production and animal welfare in Nigeria while reducing the dependence on antibiotics for successful food production. This effort is probably needed within most developing countries before efforts to control antibiotic resistance within any single country are going to prove effective.

\section{ACKNOWLEDGEMENTS}

The MacArthur Foundation provided travel support for I. O. Olatoye. This work was supported in part by the Paul G. Allen School for Global Animal Health, the Agricultural Animal Health Program, and the Agricultural Research 
Center at Washington State University, Pullman, WA.

\section{REFERENCES}

Akinyemi KO, BA Iwalokun, F Foli, K Oshodi, AO Coker (2011). Prevalence of multiple drug resistance and screening of enterotoxin (stn) gene in Salmonella enterica serovars from water sources in Lagos, Nigeria. Public Health. 125(2):65-71.

Al-Ghamdi MS, ZH Mustafa, A Al-Faky, IH El-Morsy, H Essan (2000). Residue of tetracycline compound in eastern province of Saudi Arabia. Public Health. 114:300-304.

Aypak C, A Altunsoy, N Duzgun (2009). Empiric antibiotic therapy in acute uncomplicated urinary tract infections and fluoroquinolone resistance:a prospective observational study. Ann. Clin. Microbiol. Antimicrob. 8:27.

Bonomo RA (2001). Infectious diseases and antibiotic resistance in long-term care facilities. Postgrad. Med. 109 (2 Suppl):70-77.

Cheesbrough M (2000). Microbiological test. District laboratory practice in tropical countries. Part 2. Cambridge, UK, Cambridge University Press: 434.

Cho S, Fossler CP, Diez-Gonzalez F, Wells SJ, Hedberg CW, Kaneene JB, Ruegg PL, Warnick LD, Bender JB (2007). Antimicrobial susceptibility of Shiga toxin-producing Escherichia coli isolated from organic dairy farms, conventional dairy farms and county fairs in Minnesota. Foodborne Pathog. Dis. 4:178-186

CLSI (2008). Performance standards for antimicrobial disk and dilution susceptibility tests for bacteria isolated from animals: Approved standard. 3rd Ed. CLSI document M31-A3. Wayne, PA, Clinical and Laboratory Standard Institute.

Delsol AA, J Sunderland, MJ Woodward, L Pumbwe, LJ Piddock, JM Roe (2004). Emergence of fluoroquinolone resistance in the native Campylobacter coli population of pigs exposed to enrofloxacin. J Antimicrob. Chemother. 53(5):872-874.

DHHS (2005). Enrofloxacin for poultry; Final decision on withdrawal of new animal drug application following formal evidentiary public hearing. Department of Health and Human Services, Food and Drug Administration, Center for Veterinary Medicine. Federal Register. 70(146):44105.

DHHS (2010a). Extralabel drug use in animals. Department of Health and Human Services, Food and Drug Administration, Center for Veterinary Medicine. Federal Register. 6:369.

DHHS (2010b). The judicious use of medically important antimicrobial drugs in food-producing animals. Department of Health and Human Services, Food and Drug Administration, Center for Veterinary Medicine. Federal Register. 75(124):37450-37451.

Dina OA, RO Arowolo (1991). Some considerations on veterinary drug use and supply in Nigeria. Rev. Elev. Med. Vet. Pays Trop. 44(1):2931.

Fashae K, F Ogunsola, FM Aarestrup and RS Hendriksen (2010). Antimicrobial susceptibility and serovars of Salmonella from chickens and humans in Ibadan, Nigeria. J. Infect. Dev. Ctries. 4(8):484-494.

FGN (2009). National Bureau of Statistics, Federal Government of Nigeria Factsheet, http//:www.nigeriastat.gov.ng, accessed April 14, 2010. Retrieved April 14, 2010, from http//:www.nigeriastat.gov.ng.

Fortini D, K Fashae, A Garcia-Fernandez, L Villa, A Carattoli (2011). Plasmid-mediated quinolone resistance and \{beta\}-lactamases in Escherichia coli from healthy animals from Nigeria. J. Antimicrob. Chemother. 66(6):1269-1272.

Fraunfelder FT, RL Morgan, AA Yunis (1993). Blood dyscrasias and topical ophthalmic chloramphenicol. Am. J. Ophthalmol. 115(6):812813.

French GL (2010). The continuing crisis in antibiotic resistance. Int $J$ Antimicrob. Agents. 36 Suppl 3:S3-7.

Holt-Harris JE, Teague O. (1916) A new culture medium for the isolation of Bacillus typhosa from stools. J. Infect. Dis. 18:596.

Hurd HS, MB Vaughn, D Holtkamp, J Dickson and L Warnick (2010). Quantitative risk from fluoroquinolone-resistant Salmonella and Campylobacter due to treatment of dairy heifers with enrofloxacin for bovine respiratory disease. Foodborne Pathog. Dis. 7(11):1305-1322.

Isiaka BT (1998). Livestock rearing practices and problems in Lagos. Proceeding of the Silver Anniversary Conference. Oduguwa OO,
Fanimo AO, Osinowo OA. March 21-26, 1998, Gateway Hotel, Abeokuta, Nigeria, Nigerian Society for Animal Production:223-225. Bacteroides and other genera in the human colon. Appl Environ Microbiol. 67:561-568

Kabir J, JU Umoh VJ Umoh (2004). Veterinary drug use in poultry farms and determination of antimicrobial drug residues in commercial eggs and slaughtered chicken in Kaduna State, Nigeria. Food Control. 15:3-10.

Khachatryan AR, TE Besser, DD Hancock DR, Call (2006). Use of a nonmedicated dietary supplement correlates with increased prevalence of streptomycin-sulfa-tetracycline-resistant Escherichia coli on a dairy farm. Appl. Environ. Microbiol. 72(7):4583-4588.

Khachatryan AR, DD Hancock, TE Besser, DR Call (2004). Role of calfadapted Escherichia coli in maintenance of antimicrobial drug resistance in dairy calves. Appl. Environ. Microbiol. 70(2):752-757.

Kumarasamy KK, MA Toleman, TR Walsh, J Bagaria, F Butt, R Balakrishnan, U Chaudhary, M Doumith, CG Giske, S Irfan, P Krishnan, AV Kumar, S Maharjan, S Mushtaq, T Noorie, DL Paterson, A Pearson, C Perry, R Pike, B Rao, U Ray, JB Sarma, M Sharma, E Sheridan, MA Thirunarayan, J Turton, S Upadhyay, M Warner, W Welfare, DM Livermore, N Woodford (2010). Emergence of a new antibiotic resistance mechanism in India, Pakistan, and the UK:a molecular, biological, and epidemiological study. Lancet. Infect. Dis. 10(9):597-602.

Levine M. (1918) Differentiation of $E$. coli and $A$. aerogenes on a simplified eosin-methylene blue agar. J. Infect. Dis. 23:43-47.

Li X and HH Wang (2010). Tetracycline resistance associated with commensal bacteria from representative ready-to-consume deli and restaurant foods. J. Food Prot. 73(10):1841-1848.

Mbuko IJ, MA Raji, J Ameh, L Saidu, WI Musa, PA Abdul (2009). Prevalence and seasonality of fowl typhoid disease in Zaria-Kaduna State, Nigeria. J. Bact. Res. 1:1-5.

McDermott PF, S Zhao, DD Wagner, S Simjee, RD Walker and DG White (2002). The food safety perspective of antibiotic resistance. Anim. Biotechnol. 13(1):71-84.

Mitema ES, GM Kikuvi, HC Wegener, K Stohr (2001). An assessment of antimicrobial consumption in food producing animals in Kenya. J. Vet. Pharmacol Ther. 24(6):385-390.

Nonga HE, C Simon, ED Karimuribo, RH Mdegela (2010). Assessment of antimicrobial usage and residues in commercial chicken eggs from smallholder poultry keepers in Morogoro municipality, Tanzania. Zoonoses Public Health. 57(5):339-344.

Ogbolu DO, OA Daini, A Ogunledun, AO Alli, MA Webber (2011). High levels of multidrug resistance in clinical isolates of Gram-negative pathogens from Nigeria. Int. J. Antimicrob. Agents. 37(1):62-66.

Okeke IN, A Lamikanra, R Edelman (1999). Socioeconomic and behavioral factors leading to acquired bacterial resistance to antibiotics in developing countries. Emerg. Infect. Dis. 5(1):18-27.

Oliver SP, SE Murinda and BM Jayarao (2011). Impact of antibiotic use in adult dairy cows on antimicrobial resistance of veterinary and human pathogens: a comprehensive review. Foodborne Pathog. Dis. 8(3):337-355

Owoade AA, DO Oluwayelu, OA Fagbohun, W Ammerlaan, MN Mulders and CP Muller (2004). Serologic evidence of chicken infectious anemia in commercial chicken flocks in southwest Nigeria. Avian Dis. 48(1):202-205.

Pallecchi L, A Bartoloni, C Fiorelli, A Mantella, T Di Maggio, H Gamboa, E Gotuzzo, G Kronvall, F Paradisi, GM Rossolini (2007). Rapid dissemination and diversity of CTX-M extended-spectrum betalactamase genes in commensal Escherichia coli isolates from healthy children from low-resource settings in Latin America. Antimicrob. Agents Chemother. 51(8):2720-2725.

Patel U, R Kirby (2008). Infections after prostate biopsy and antibiotic resistance. BJU Int. 101(10):1201-1202.

Riviere JE, JW Spoo (1995). Chemical residues in tissues of food animals. Veterinary Pharmacology and Therapeutics. HR Adams. Ames, IA, lowa State University Press:1148-1115.

Sato K, Bartlett PC, Saeed MA (2005). Antimicrobial susceptibility of Escherichia coli isolates from dairy farms using organic versus conventional production methods. J. Am. Vet. Med. Assoc. 226:589594

Shoemaker NBH, Vlamakis KH, Salyers AA (2001). Evidence for 
extensive resistance gene transfer among Bacteroides spp. and among Bacteroides and other genera in the human colon. Appl.

Environ. Microbiol. 67:561-568

Simonsen GS, JW Tapsall, B Allegranzi, EA Talbot and S Lazzari (2004). The antimicrobial resistance containment and surveillance approach-a public health tool. Bull World Health Organ. 82(12):928934.

Singer RS, Ward MP, Maldonado G (2006). Can landscape ecology untangle the complexity of antibiotic resistance? Nat. Rev. Micro. 4:943-952.

van Eekeren N, A Maas, HW Saatkamp and M Verschuur (2004). Small-scale poultry production in the tropics. Wageningen, Netherlands, Agromisa Foundation.
Venkateswaran K, A Murakoshi and M Satake (1996). Comparison of commercially available kits with standard methods for the detection of coliforms and Escherichia coli in foods. Appl Environ Microbiol. 62(7):2236-2243.

Young NS, BP Alter (1994). Epidemiology of acquired aplastic anemia. Aplastic anemia acquired and inherited. Philadelphia, W.S. Saunders:24-31. 